\title{
Analysis of Contemporary College Students' Physical Exercise Behavior under the NCP
}

\author{
Yuefeng Jin \\ PE Department, Shanghai Sanda University, Shanghai, China
}

\begin{abstract}
Objectives: Facing the rapid spread of NCP in the world, it is particularly important to improve students' immunity and resistance through physical exercise.Methods: This paper investigates the physical exercise behavior of college students by means of literature investigation, expert interview, questionnaire survey and behavior analysis.Results: Under the NCP, there are individual differences in the enthusiasm of college students to participate in physical exercise; the self-efficacy of male and female students participating in physical exercise is low, and girls are lower than boys; boys' expectation of results is higher than girls'; the self-adjustment ability of boys is higher than that of girls. There is a negative correlation between students' participation perception barrier and participation enthusiasm. Conclusions:Home exercise is training for the public during the NCP and special weather conditions. By creating a strong atmosphere of physical exercise and sports culture, vigorously publicizing and popularizing sports knowledge and exercise effects, carrying out personalized physical education, reducing perceptual barriers and adjusting physical exercise plans, students' enthusiasm for participating in physical exercise is mobilized to improve students' physical health.
\end{abstract}

Keywords: College students, Social cognitive theory, Physical exercise, Behavioral analysis

\section{I.Background}

The rapid spread of NPC in the world has made many people threatened by COVID-19. The strength of individual immunity directly determines whether COVID-19 will invade your body. After the epidemic, everyone has begun to realize the importance of physical exercise, and the goal of physical exercise has begun to enter the development stage of "Health Promotion" from "Strengthen Physical Fitness"[1]. In the middle and late last century, Bandura, a famous American psychologist, put forward the educational theory of social cognition. After his theory was put forward, it began to develop rapidly in the world at the end of last century. Bandura pointed out that individual behavior will not only be controlled and regulated by personal psychological factors, such as physiological conditions, cognition and emotions, interests and hobbies, etc. It will also be affected by external environmental factors, such as social factors, environmental factors, family atmosphere, etc. From our analysis of social cognition theory, we can see that there are four influencing factors of social cognition on individual participation in physical exercise, which are analyzed in depth below.

\section{II.Influencing Factorsof Social Cognitive Theoryon Students' PhysicalExercise}

\subsection{Result expectation}

The result expectation is the student's speculation on the results caused and caused by personal behavior in personal life, participation in study or sports and other activities[2]. In short, it means that one predicts whether one's behavior can achieve what kind of results. If one feels that one can achieve that kind of result, one will generate a kind of motivation to do it. If you feel that you can't achieve that result, you will resist or retreat psychologically. When the NCPwas first launched in 2020, the prediction of the development trend of the new crown pneumonia epidemic is still very optimistic. COVID-19's spread has not attracted enough attention. But as the gap between reality and forecast results is getting bigger and bigger, people are beginning to be in tension and

ISSN: 0010-8189

(C) CONVERTER 2021

www.converter-magazine.info 
uneasiness. The NCPis a new disease. People are beginning to realize the importance of physical exercise to health and enhance their resistance.

\subsection{Self-efficacy}

Judgment is the essence of self-efficacy, and it is the personal judgment under the interaction between human beings and their environment. When self-efficacy is strong, individuals can have greater interest in new things or problems around them, and mobilize their energy and time to solve problems. When these people meet difficulties and are on the way to overcome them, their self-efficacy will be enhanced and strengthened. However, for people with weak self-efficacy, when they encounter difficulties and problems, they always doubt their personal ability and tend to take an evasive attitude towards things. Bandura pointed out that there are four aspects of information in people's participation in social activities that can promote people to form a sense of self-efficacy.

Personal self-perception in activities, such as successful experience in life, study and work, can enhance personal self-efficacy in social activities. On the contrary, the lessons of failure will reduce people's self-efficacy in activities[3]. The self-efficacy of alternative experience type means that when people find people with the same ability as themselves, they observe their activity achievements and finally convince themselves that they can achieve the same effect in similar activities under the same conditions. They mainly reduce or enhance their selfenergy efficiency by observing the activity results of others. Verbal persuasion, as its name implies, is the appreciative and encouraging language given by individuals or others when solving tasks, which can enhance people's self-efficacy when solving problems. Generally, this kind of persuasive language needs to be consistent with the actual situation. Using affirmation and positive encouragement can mobilize the enthusiasm of individuals. However, persuasion language that is inconsistent with reality will inhibit people's personal enthusiasm when participating in activities[4]. In addition, self-efficacy is also affected by personal physical and mental state, For example, when people carry out a certain job, positive mentality and emotion can increase their ability to solve problems and improve their sense of self-efficacy. On the contrary, negative mentality and emotion will lead to the limitation of personal ability, thus causing them to feel frustrated and their sense of self-efficacy to decrease. The research on this point shows that optimism is an effective help for students to study and participate in sports activities, and students should cultivate a more optimistic and positive attitude. As far as individual selfefficacy is concerned, the training environment is the social environment in which they live. Therefore, in higher education, the school should pay attention to the guidance of students, so that they can face difficulties with a positive attitude, strengthen their sense of self-efficacy and cultivate their abilities after overcoming difficulties.

\subsection{Perceived obstacles}

The perceived obstacle, as its name implies, is a kind of behavioral cognition generated by students when learning new things or solving difficult problems, feeling that what they have learned is beyond the scope of their personal abilities[5]. For example, before students with poor physical quality participate in intensive physical exercise activities such as long-distance running, they have an understanding that they cannot complete and are difficult to complete after measuring the difficulty of exercise, personal physical quality and willpower. The COVID-19 is a new virus. People feel a sense of disturbance onNCP, which leads to confusion in the exercise of the new crown pneumonia. Later, the whole country solved this problem through online physical education, home physical exercise, video physical exercise guidance and other ways, and the students' exercise behavior was not affected.

\subsection{Self-adjusting behavior}

Self-adjustment behavior is a dynamic mechanism for individuals to develop from unbalanced to balance in cognitive development[6]. From a broad perspective, it is a process in which individuals weaken or strengthen their behavior with rewards and punishment measures that individuals can achieve when setting standards for their own behavior. In a narrow sense, it is a process of maintaining and enhancing personal behavior by using continuous rewards when individuals make continuous efforts and achieve the goals set by individuals.

ISSN: 0010-8189

(C) CONVERTER 2021

www.converter-magazine.info 


\section{III.Waysto Analyze College Students' Physical ExerciseBehavior}

\subsection{Analysis method}

In order to analyze the physical exercise behavior of college students under the social cognitive theory, we should make use of the above four influencing factors to formulate analysis methods, and finally clarify the physical exercise status of students through the performance of students. The analysis shows that when students' selfadjustment behavior, self-efficacy and expected results are higher, it indicates that students' personal enthusiasm and possibility of participating in physical exercise are higher. In addition, when the obstacles perceived by students are less, the willingness and possibility of students to participate in physical exercise are also higher.

\subsection{Suggestions for higher education from the perspective of analysis methods}

Through the investigation of college students, it can be seen that there are individual differences in the enthusiasm of individuals to participate in physical exercise, regardless of male or female students in colleges and universities. Therefore, in higher education, colleges and universities can change students' social cognitive theory, so as to improve students' self-adjustment behavior, self-efficacy and other aspects, reduce students' perceived exercise obstacles, and improve students' personal enthusiasm to participate in physical exercise. When the physical education teachers in colleges and universities carry out physical education teaching, It is necessary to make use of the decomposition and demonstration of sports movements to reduce the difficulty of physical exercise intuitively observed by students, so as to improve students' sense of self-energy efficiency in participating in sports activities, fully reduce their perceived exercise obstacles, enable them to actively participate in physical exercise, and strengthen their physical quality[7]. In addition, Bandura pointed out that students can learn new skills or correct certain behavioral characteristics by observing and analyzing other people's behaviors and results. Under the more intuitive demonstration teaching, I believe it can have a positive effect on students and make them strengthen their confidence in physical exercise. Finally, teachers should be able to pay attention to the emotion expressed by individuals in teaching, Treat all students equally, encourage and praise students' learning achievements and processes in time, realize the purpose of strengthening students' participation motivation, let all students get greater satisfaction and sense of accomplishment in physical exercise, and finally realize the growth of different students' sports interests, laying a foundation for students' physical enhancement.

\section{The Resultsof Analyzing thePhysical Exercise Behaviorof College Students}

\subsection{Analysis of students' self-efficacy in physical exercise}

Through analysis and research, it can be seen that the self-efficacy of male and female students is low in physical exercise, and the female students are relatively lower than the male students. For girls, it is easier for them to use the interface to avoid physical exercise, such as unaccompanied, weather obstacles, etc., which will become excuses for students to refuse to participate in exercise. For boys, when they are resisted by others and unaccompanied, their self-efficacy is higher, which indicates that students' self-efficacy in physical exercise is less affected by resistance from others or unaccompanied. However, on the other hand, girls' enthusiasm for participating in physical exercise will decline relatively under the condition of being blocked or unaccompanied. In addition, under the influence of weather factors, college students' self-efficacy is weak regardless of gender, which indicates that such factors have a greater impact on students' exercise[8]. The factors affecting the NCP have great influence on students' exercise. During the NCP, college students' physical exercise self-efficacy decreased significantly.

After the above analysis, we can see that in order to promote the enthusiasm of college students for exercise, colleges and universities should learn the concept of quality education, use sports teaching materials that meet students' different needs and sports preferences, and ensure that they can meet students' professional physical needs to carry out physical education, so as to strengthen the educational function of physical education. When colleges ISSN: 0010-8189 
and universities carry out physical education, they should not only pay attention to the improvement of students' physique, but also cultivate students' interest in sports and exercise enthusiasm, so as to gradually make students cultivate good personal sports habits. Colleges and universities can combine students' psychological needs, organize diversified and interesting sports competitions in schools, make use of the mechanism of multi-level leagues, create a stronger atmosphere and culture of physical exercise in schools, and do a good job in popularizing all kinds of sports knowledge in conjunction with schools and hospitals, so that students can fully realize the benefits brought by participating in exercise. Finally, in the publicity of epidemic prevention and control, it is also necessary to increase suggestions on individual scientific and reasonable physical activities, including location, frequency and exercise intensity, so as to help the public exercise more scientifically and improve their immunity.

\subsection{Analysis of expectation of results in students' physical exercise}

The goal of physical exercise is to help students strengthen their cardiopulmonary ability and achieve the effects of preventing diseases and shaping their bodies[9]. In the analysis and research, both male and female college students are more positive in the expectation of results. But relatively speaking, boys expect to strengthen their strength more, while girls have a negative attitude towards it. In terms of psychological expectation results, male and female students have positive expectations on ability display, self-confidence improvement or charm growth, while male students are more positive than female students in relieving stress expectation.

The analysis of students' expectation of results, It reflects students' value expectation in participating in exercise, Therefore, in order to ensure that college students can actively and independently participate in physical exercise, colleges and universities can vigorously publicize sports and exercise effects, so that students can fully realize the value and function brought by participating in exercise, and then cultivate personal reasonable exercise habits and gradually cultivate lifelong physical exercise awareness. In the propaganda work, colleges and universities can combine the campus environment with online propaganda. In online propaganda, the official Web Chat official account on campus is used to explain videos and various related sports contents for students' knowledge and skills of popular science physical exercise. Combine with the NCP knowledge was publicized, The public awareness of new crown pneumonia was strengthened. Different publicity methods and different publicity channels were used to publicize basic knowledge of new crown pneumonia. The public was reminded to update their knowledge of new crown pneumonia in a timely manner and to guide students to take exercise during the NCP and improve their health.

\subsection{Analysis of students' self-adjustment behavior in physical exercise}

According to the analysis and investigation, the self-adjustment ability of boys is relatively higher than that of girls. In the established goals, the physical exercise goals of male and female students are relatively vague and unclear, and the adjustment ability of male and female students is better in the plan adjustment. Therefore, it can be seen that the established goals or plan adjustments set by students to participate in physical exercise will affect students' enthusiasm for participating in exercise. Due to the NCP, the teaching methods of university physical education curriculum have changed, resulting in a significant impact on the time and intensity of physical exercisee[10].

Some scholars pointed out when studying the participation of young people in physical exercise in China that for young people, their personal enthusiasm for participating in sports activities will be affected not only by the sports skills mastered and understood by individuals, but also by their expectations for physical development[11]. Therefore, it is necessary to ensure the further development of physical education in colleges and universities and fully improve students' exercise autonomy. While doing a good job in skill teaching, colleges and universities should also be able to build departments that manage students' physical health, such as school hospitals, physical education departments and so on. All departments should be able to work as a team, sharing health resources, and put forward effective exercise guidance for students in time, so that they can set effective and reasonable exercise goals according to their actual situation, or adjust their exercise plans, and carry out personalized and customized physical education, so that students can exercise scientifically, finally feel the benefits brought by physical exercise,

ISSN: 0010-8189

(C) CONVERTER 2021

www.converter-magazine.info 
and cultivate good sports habits.

\subsection{Analysis of obstacles perceived by students in physical exercise}

The obstacles to participation perceived by students in physical exercise are generally negatively correlated with students' enthusiasm for participation, that is, the greater the obstacles perceived by students, the smaller the students' enthusiasm for participation will be and the more negative it will be[12]. The NCP is a new type of pneumonia. The results show that the effect of online physical education on College Students' physical and mental health is not important, and the attraction to students is not enough. Eventually, students lose or reduce interest in physical exercise. Therefore, PE teachers need to strengthen the monitoring of online physical education teaching quality.

Therefore, it is necessary to do a good job in college education, so that more college students can keep their enthusiasm for physical exercise, and colleges and universities should make use of various ways to fully reduce students' cognitive barriers in participating in sports activities. In the modern living environment, colleges and universities can use Internet technology and mobile communication to achieve this goal. For example, the corresponding functions, usage methods, technical training or health care and rehabilitation of each equipment can be provided to each student on and around sports equipment by means of two-dimensional code scanning and identification. For example, colleges and universities can paste two-dimensional codes at football field goalposts, table tennis tabletops and other positions. When students carry out physical exercise, they can directly use mobile devices to scan codes and directly obtain information about equipment and sports. In the processing of information materials, colleges and universities can try their best to make use of simple cartoons with pictures, texts and videos, which are more intuitive and interesting. Let students use the network and mobile devices to carry out direct and repetitive skills learning, and then carry out physical exercise in a standardized and safe way. In addition, in the corridors of teaching buildings, library desktops and other places where students often contact, Two-dimensional codes can also be pasted in schools. Two-dimensional codes covering physical exercise knowledge of whole body activities can be pasted in corridors, and two-dimensional codes related to relaxing cervical vertebrae and eye exercises can be pasted on the desktop of libraries. Interesting design of two-dimensional codes can be done to enhance students' interest in scanning codes.

\section{Conclusion}

After the outbreak of the NCP, different media have conveyed the outbreak of the outbreak, which enables more people to realize the importance of physical exercise. In other words, the epidemic makes people's physical exercise more positive. However, the survey data show that the attitude of physical exercise is not positive enough[13].In combination with the above, colleges and universities is one of the important bases for training professional talents for our country. In college education, colleges and universities should not only attach importance to the learning of students' professional skills, but also attach importance to the improvement of students' physical quality. Physical exercise is an effective way to promote students to strengthen their physique, but there are great differences in the enthusiasm of college students to participate in physical exercise[14]. Colleges and universities should be able to use social cognitive theory to conduct scientific and sufficient analysis and research on students' exercise behavior, start from the analysis results to clarify various factors that affect students' participation in physical exercise, and then formulate corresponding teaching methods and strategies to provide students with a sufficient environment for participating in exercise.

Through the analysis of students' exercise behavior, it can be seen that in order to ensure the growth of students' exercise autonomy and enthusiasm, colleges and universities should strengthen students' self-efficacy and selfadjustment behavior through different ways, and then fully reduce the obstacles perceived by students in physical exercise. Through the construction of sports atmosphere, more required sports knowledge will be popularized for students, so that students can feel the benefits brought by participating in sports activities, and the stadiums and gymnasiums will be opened according to the actual situation. In addition, in the modern living environment,

ISSN: 0010-8189

(C) CONVERTER 2021

WWW.converter-magazine.info 
colleges and universities can combine physical education, sports publicity with new media publicity, information technology and other aspects to reduce perceptual barriers for more students, help students adjust their exercise plans, and finally ensure the good development of physical education in colleges and universities.

\section{References}

[1] Y.C.Liang,Q.Zhang, "Urban residents' physical exercise mode and collective social capital-a perspective of heterogeneous social space," Journal of Shanghai Institute of Physical Education, vol. 44, no. 4, pp. 12-22, 2020.

[2] M.Lei. "How physical exercise promotes happiness-on the intermediary role of social capital," Journal of Shanghai Institute of Physical Education, vol. 44, no. 4, pp. 23-30, 2020.

[3] X.H. Wang, C. Peng,"Study on the influence mechanism of socio-economic status of the elderly on health-also on the mediating effect of lifestyle, public service and social psychology," China Health Policy Research, vol. 13, no. 3, pp. 21-30, 2020.

[4] B.L.Dong, L.J. Mao, “The influence of adaptive perfectionism and autonomous motivation on adolescent exercise persistence: the moderating effect of parental autonomous support," Journal of Shanghai Institute of Physical Education, vol. 44, no. 2, pp. 11-21, 2020.

[5] D.Q. Zhang, S.T. Che, Y.Z. Ke, et al.,"Characteristics and Enlightenment of Training Mode for Students Majoring in Physical Education in American Universities-Taking Wyoming University as an Example," Sports Culture Guide, no. 2, pp. 103-110, 2020.

[6] L. Liu, "Effect of community group aerobic training on physical and mental health and cognitive function of the elderly," Journal of Southwest Normal University (natural science edition), vol. 45, no. 2, pp. 113-122, 2020.

[7] Z.J. Zhang, Y.Q. Miao,"Study on the contribution of basic public health services to residents' health differences," Chinese Population Science, no. 1, pp. 78-89 +127-128, 2020.

[8] C.R. Yang, Y. Wang, X. Chen, "Investigation on physical exercise behavior of community residents in Guangzhou-based on the perspective of social stratification," Journal of Guangzhou Institute of Physical Education, vol. 40, no. 1, pp. 23-26, 2020.

[9] H.M. Zhong, Z.H.Wang, Y. Gao, "Can taking part in physical exercise improve social and economic status? --Empirical research based on CGSS2015 survey data," Journal of Wuhan Institute of Physical Education, vol. 54, no. 1, pp. 38-46, 2020.

[10] L. Luo, X.J.Ceng, X. Liao, et al.,"Public health knowledge, coping styles and exercise behavior during the NCP outbreak," China Public Health, vol. 36, no. 2, pp. 156-159, 2020.

[11] S.M. Wang, Y. Cao, "Report on the development of positive psychological benefits scale for middle school students' physical exercise,” Physical Education Research, vol. 2, no. 6, pp. 47-56, 2019.

[12] W. Lu, H.B. He."Research on internalization mechanism and behavior promotion of college students' exercise motivation,” Journal of Xi'an Institute of Physical Education, vol. 37, no. 1, pp. 98-106, 2020.

[13] T.P. Wei, L. Chen, “NCP background, college students' exercise attitude, exercise behavior and physical self-esteem," Sports Research, vol. 41, no. 04, pp. 38-42, 2020.

[14] C.L. Gan, S. Guo, Y.F. Zhang,"Study on the relationship between social and economic status and physical fitness level of Macao citizens," China Sports Science and Technology, vol. 55, no. 12, pp. 22$30+38,2019$.

ISSN: 0010-8189 